Thomas Schlag

\title{
Gespeichert, erinnert, vergessen? - Digitale Speicherdynamiken und ihre Bedeutung für eine menschenwürdige Erinnerungs- und Bildungskultur
}

\section{Phänomene und Probleme digitaler Speicherdynamiken}

Die Möglichkeiten digitaler Speicherung sind inzwischen fast unbegrenzt. Auf Datenträgern unterschiedlichster Art, etwa in Clouds und digitalen Repositorien, werden nicht nur alltägliche Informationen gesammelt, sondern wesentliche Erinnerungsstücke und Geheimnisse, manchmal ganze Biografien des individuellen Lebens abgelegt. Digitale Speichermedien ermöglichen dauerhafte Erinnerung. Sie halten Wesentliches und Wichtiges für die Nachwelt fest -, aber dies keineswegs nur durch und für die gespeicherte Person selbst. Digitale Speicherungsmöglichkeiten sind höchst ambivalent. Sie machen die Möglichkeiten der Selbstbestimmung darüber, was erinnert und was vergessen werden soll, schwieriger. Die Spuren von Lebensläufen werden laufend aufgezeichnet, ohne dass die Nutzer*innen im Einzelnen wissen, von wem, nach welchen Kriterien und mit welchen kurz-, mittel- oder langfristigen Absichten dies geschieht.

Daraus ergeben sich eine Reihe grundsätzlicher Fragehorizonte und Probleme, auf die es sich auch in theologischer Perspektive näher einzugehen lohnt. Dass diese Problemlagen im Folgenden immer wieder in Frageform gefasst werden, hat mit der unüberschaubaren und mithin unübersichtlichen Entwicklungsdynamik digitaler Medien selbst zu tun: Es ist gegenwärtig noch kaum absehbar, wohin sich die digitale Speichernutzung bzw. Nutzungsspeicherung entwickelt. Hier sind viele Zukunftsszenarien entweder ins Gewand bloßer Spekulation oder generalisierender Drohbotschaft gekleidet, was die sachliche Debatte nicht unbedingt befördert. Insofern erscheint es an der Zeit, prophylaktisch die wesentlichen Fragen, die sich mit der Speicherung „unserer“ Daten verbinden, einmal näher zu identifizieren. Noch scheinen gewisse Einflussmöglichkeiten auf diese Entwicklungen zu bestehen, bevor diese ganz und gar unkontrollierbare Eigendynamiken annehmen. 


\section{Speichertechniken:}

Das geringste Ambivalenzproblem dürfte die technische Seite darstellen. In technischer Hinsicht ist tatsächlich nicht klar, ob das Gespeicherte in Jahrzehnten wirklich noch abgerufen werden kann. Möglicherweise können die jetzt gespeicherten Informationen gar nicht mehr gelesen werden, weil die jetzigen Lesegeräte dann schlichtweg veraltet sind oder die Festplatten über die Jahrzehnte hinweg ihren Geist aufgegeben haben werden. Dass analoge archivalische Nachlässe aus Papier und Dokumenten unter Umständen eine erheblich längere Bestandsdauer haben als digitale Formate, wissen Archivare so eindrücklich wie leidvoll zu berichten. In diesem Sinn stellt die Vergänglichkeit bestehender Speichersysteme durchaus eine Gefahr für die Wahrung des kollektiven Gedächtnisses dar. Stewart Brand beschwor bereits 1999 die Gefahr eines „Digital Dark Age“ unter Verweis auf die kurze Haltbarkeit digitaler Datenträger (Brand 1999). 2015 prägte der US-Amerikaner Vinton G. Cerf den Ausdruck der „Digital Black Holes“ als Metapher für das Risiko, alle digital gespeicherten Inhalte für immer zu verlieren. Der Internet-Pionier warnt vor der Obsoleszenz von Programmen und Daten aus den Anfangszeiten der Digitalisierung und rät mit Blick auf das wirklich Wichtige: „Druckt es aus, nehmt es auf oder kauft es auf Vinyl“ (Arte 2018).

Für die theologische Sicht ist interessant, dass ein Silicon-Valley-Projekt unter dem Titel Arch Mission angesichts dieser Problematik das Ziel hat, Erkenntnisse über neuartige Speichermöglichkeiten zusammenzuführen und dadurch wichtige Werke zu archivieren sowie den Wissensschatz der menschlichen Spezies zu bewahren. Mithilfe von Quarzglas, das in fünf Dimensionen von einem Laser beschrieben wird, erstellt die Stiftung sogenannte Arch Libraries, die die Zeit überdauern sollen wie die Tiere der Arche Noah die Flut - wobei ironischerweise die auf den Mond gesandte digitale Lunar Library dort im Mai 2019 vermutlich beim Aufprall zerstört wurde (Arch Mission Foundation 2020). Aber wie gesagt, diese technischen Probleme der Speicherung sind vergleichsweise harmlos.

\section{Speicherzugänge:}

Wesentliche Speicherdaten werden anhand technischer Codierung verborgen und sind eigentlich nur für diejenigen zugänglich, die den Zugangscode besitzen. Aber wo ein digitales Schloss ist, ist in jedem Fall ein Schlüssel vorhanden. „Besitzen“ ist nicht mehr exklusiv auf der Seite desjenigen, der über den Code verfügt. Das, was wir „privat“ von uns speichern, 
ist prinzipiell auch für andere zugänglich und vermutlich irgendwann auch für diejenigen greifbar, die jetzt noch nicht über den passenden Schlüssel verfügen. Insofern stellen sich hier folgende Fragen: Wer hat Zugriff auf die Erinnerungen, wer nutzt sie heute oder in Zukunft, um dokumentiertes Leben aufzurufen? Wer greift schon jetzt auf die produzierten Daten, also auf die je individuelle Geschichte zurück, und zu welchem Zweck geschieht dies? Wer sind - schon jetzt und in Zukunft - die Eigentümer*innen der individuellen und kollektiven Erinnerungswolken?

\section{Speichernde Autoritäten:}

Die Frage nach den Speichermöglichkeiten und -zugängen wirft die weitergehende Problematik speichernder Autoritäten auf. Anhand bestimmter Algorithmen werden längst bestimmte Bilder, Profile und Identitäten eines Menschen erstellt. Hier greifen Selektionsmechanismen, deren innere Logik und Kriterienbildung keineswegs automatisch offengelegt werden. Die Speicherautoritäten entziehen sich zunehmend der Kontrolle, was nicht erst dann problematisch wird, wenn sie sich mit bestimmten konsumorientierten oder auch politischen Absichten verbinden.

Es steht jedenfalls zu vermuten, dass wir schon längst nicht mehr Herr im eigenen Haus der Erinnerung darüber sind, was von uns gewusst und als wesentlich selektiert wird. Der Computer- und Medienentwickler und einer der später schärfsten Digitalisierungskritiker, Joseph Weizenbaum, konstatiert zur Frage digitaler Autoritäten in wiederum theologischer Metaphorik: „Ich glaube wirklich, dass die Naturwissenschaft ... heute alle Merkmale einer organisierten Religion hat. Da gibt es Novizen, das sind die Studenten an Universitäten. Da gibt es Priester, das sind die jungen Professoren, dann gibt es die Monsignori, das sind die älteren. Es gibt Bischöfe und Kardinäle. Es gibt Kirchen und es gibt Kathedralen. Meine eigene Universität, das Massachusetts Institute of Technology, ist eine Kathedrale innerhalb der Naturwissenschaft. Es gibt sogar Päpste und - das ist sehr wichtig - es gibt Häretiker. ... Und dann gibt es die große Masse der Gläubigen. So betrachtet besteht überhaupt kein Unterschied zwischen dem Naturwissenschaftsglauben und dem Glauben an die Lehre der katholischen Kirche im Mittelalter" (Weizenbaum 2006, 166f.).

So ist zu fragen: Wer zeichnet welches Bild von uns, ganz abgesehen davon, dass die je eigene Biographie möglicherweise aus bestimmten Interessen "gefakt" wird? Von erheblicher juristischer Tragweite ist die Frage: Wer wird am Ende bzw. nach unserem Ableben darüber entscheiden, was erhalten bleiben soll und was gelöscht wird (zu den erbrechtlichen Aspekten 
vgl. Herzog 2018)? Können Daten unsterblich sein, d. h. dem Vergessen gar nicht anheimfallen? Ist aber die Gefahr, dass andere mit Hilfe einer bestimmten Datenselektion das Leben eines Individuums interpretieren, der Preis für digital garantierte Ewigkeit?

Die Thematik der Speicherautoritäten trägt dabei nicht nur eine individuelle, sondern auch eine kollektive Dimension, nämlich die Erzeugung bestimmter Bilder von Geschichte, in sich. Es geht um die Frage, wer zukünftig darüber bestimmt, was als wesentliche Daten, Ereignisse oder Verläufe von Geschichte gelten soll. Wer bestimmt darüber, woran sich eine Gesellschaft hinsichtlich der eigenen Genese erinnern soll? Erinnerungen sind immer Konstruktionen bestimmter Autoritäten, die in der Regel nicht interesselos sind: "Who controls the past, ... controls the future: who controls the present controls the past' "(Orwell 1949, 37). Die Spannung zwischen dem Faktum speichernder Autoritäten und der freien Selbstbestimmung des Menschen bzw. der freien Deutung über die Genese der Gesellschaft ist unverkennbar.

Man könnte nun diese Frage für unerheblich halten, da ohnehin alles gespeichert wird. Aber gerade deshalb werden zukünftig Selektionen über das zu Löschende und das Erhaltenswerte stattfinden. Wer werden dann die Autoritäten sein, die die entscheidenden Auswahlkriterien treffen? Nach welchen politischen und ideologischen Kriterien werden die entsprechenden Entscheidungen vorgenommen werden? Und werden diese überhaupt noch in demokratischem Sinn transparent, partizipativ und revidierbar sein?

So kann in Hinsicht auf Speicherautoritäten die paradoxe Situation entstehen, dass Erinnerung von Instanzen gesteuert wird, die sich jeglicher Machtkontrolle systematisch entziehen. Bei diesen Autoritäten ist zum einen an private Unternehmen zu denken, die ihre Speichervorgänge und -inhalte aus unternehmensstrategischen Gründen bewusst dem öffentlichen, kontrollierenden Zugriff entziehen. Zum anderen ist an private „Open source“-Autoritäten zu denken, die zwar öffentlich präsent, aber kaum kontrollierbar sind. Mit anderen Worten: Sowohl verheimlichte wie veröffentlichte Erinnerungen entziehen sich in gewissem Sinn der Kontrolle. Insofern entstehen aufgrund der digitalen Speichermöglichkeiten sowohl hinsichtlich unternehmerischer wie auch privater Autoritäten eminent politische und rechtliche Problemlagen.

Dass sich diese Grundfragen inzwischen auch in handfesten politischen Forderungen manifestieren, macht die in einem seit 2016 stattfindenden Diskussionsprozess entwickelte sogenannte Charta der Digitalen Grundrechte der Europäischen Union deutlich. In dieser heißt es zur Thematik unter Artikel 7 (Privatsphäre, Vertraulichkeit und Datenschutz): „(1) Jeder 
Mensch hat das Recht auf den Schutz seiner Daten und die Achtung seiner Privatsphäre. (2) Personenbezogene Daten dürfen nur nach Treu und Glauben für festgelegte Zwecke beim Betroffenen erhoben und verarbeitet werden, wenn hierfür eine gesetzliche Grundlage besteht. Die Datenverarbeitung muss sicher, fair, transparent und nach dem Stand der Technik gestaltet werden. (3) Rechte auf Löschung, Berichtigung, Widerspruch, Information und Auskunft sind zu gewährleisten. (4) Jeder Mensch hat das Recht auf digitalen Neuanfang. Dieses Recht findet seine Grenzen in den berechtigten Informationsinteressen der Öffentlichkeit" (Charta der Digitalen Grundrechte 2018).

\section{Gespeicherte Identität:}

Die Möglichkeiten digitaler Archivierung könnten dazu führen, dass zwar alles aufbewahrt werden kann, sich aber wesentliche Bestandteile der persönlichen und kollektiven Erinnerung und Identität ins Wolkige verflüchtigen und gegenstandslos werden. Braucht Erinnerung, so ist zu fragen, nicht das Greifbare, Sichtbare und Lokale, die wirkliche Anschauung dessen, was man in Tagebüchern und Briefen, in Fotoalben oder im eigenen Bücherregal real und mit Patina belegt vor Augen hat? Was wird aus den haptischen und olfaktorischen Eindrücken, wenn alles nur noch eindimensional auf dem Monitor sichtbar ist? Mag man sich Festplatten tatsächlich als begehbare „lieux de mémoire“ (Nora 1989) vorstellen? Ersetzen digitale Speichermedien am Ende das kollektive und kulturelle Gedächtnis, weil sie anschaulich-aktives Erinnern und Vergessen unnötig oder unmöglich machen? Und wäre dies dann die „Erfüllung“ der Luhmann'schen Befürchtung, dass gerade die Tendenz, alles erinnern zu können, die größte Gefahr für die Erinnerung selbst darstellt?

Die Frage nach gespeicherter Identität umfasst noch eine viel weiterreichende existenzielle Dimension, insofern die Frage der Verfügbarkeit erhebliche Konsequenzen für die Kernbestimmung des Individuums mit sich bringt. Zu beachten ist hier, ob die je "gespeicherte Person“ im Einzelfall überhaupt noch die Chance auf einen Neuanfang (vgl. Arendt 1994, 276) und auf die Neubestimmung der eigenen Identität hat, wenn bestimmte Erinnerungen über sie ohne eigene Verfügung gestreut werden oder ob sie über das Skandalöse, das von ihr dokumentiert ist, auf ewig definiert bleibt. Im schlimmsten Fall wird man von Autoritäten, die man nicht einmal kennt, geschweige denn aktiv kontaktieren kann, fremdbestimmt. In diesem Sinn ist die digitale Speicherwelt tatsächlich eine "Global village“ (McLuhan 1962), die über ganze Generationen hinweg nicht vergisst und 
nicht vergibt - mit dem Unterschied, dass sich der in der digitalen Welt manifestierte Leumund endgültig nicht mehr aus der Welt schaffen lässt.

Diese existenziell relevante Verfügbarkeits- bzw. Verfügungsproblematik wird noch prekärer, seitdem es möglich ist, die individuelle digitale Identität auch dann noch weiterzuentwickeln, wenn die einzelne Person längst gestorben ist. Entsprechende Programme wie etwa MyLifeBits oder LifeLog (vgl. Zierold 2008, 175) oder replika.ai können das individuelle Erinnerungsmaterial kreativ so ansammeln und auch weiterentwickeln, dass die digital gespeicherte Identität durch neue Fütterung (etwa durch weitere Informationen und kommunikative Interaktionen) ständig erweitert wird und damit die Replikant*innen den Status digitaler Unsterblichkeit erlangen (vgl. Newton, o.J.). Beantwortet „am Ende“ womöglich die Cloud des Homo Deus die uralte Menschheitssuche nach Unsterblichkeit so, dass neue gottähnliche Verfügbarkeiten an die Stelle des Unverfügbaren treten?

Der Digitalkritiker Jaron Lanier vermerkt dazu durchaus erstaunt: „Google hat bekanntlich ein Projekt zur ,Lösung des Todes' finanziert. Dies ist so präzise eine religiöse Behauptung, dass ich überrascht bin, dass die Religionen der Welt gegenüber Google keine Klage mit Hinweis auf Urheberrechtsverletzungen eingereicht haben" (Lanier 2018, 133). Wird somit mit „uns“ auf der Grundlage dessen, was von uns einst gespeichert wurde, weiterkommuniziert?

Viele dieser Fragen könnten uns im Prinzip herzlich egal sein. Denn wenn in einer solchen Weise über unsere Erinnerungen und über uns als Person verfügt werden sollte, sind wir selbst längst nicht mehr unter den Lebenden. Und doch verschärfen diese technischen Speichermöglichkeiten die Frage der Bestimmung über die eigene Identität schon zu Lebzeiten. So ist wiederum noch vertiefter zu fragen: Haben wir ein Anrecht darauf zu wissen, was mit uns nach unserem leiblichen Tod geschieht? Gibt es so etwas wie postmortale Selbstbestimmung? Tragen die individuellen Erinnerungen eine Würde in sich, selbst wenn die betroffenen würdevollen Einzelwesen nicht mehr sind? Wem soll jetzt schon die Deutung des irgendwann vergehenden Lebens anvertraut werden?

Nun könnte man einwenden, dass die Frage des Speicherns und Erinnerns nichts Neues unter der Sonne ist. Und doch zeigt sich hier, so meine These, eine neue Qualität, die darin besteht, dass aufgrund der technischen Speichermöglichkeiten sowohl hinsichtlich der persönlichen Identitätsbestimmung wie auch der weiterreichenden kollektiven Erinnerungskultur erhebliche dynamische Fremdsteuerungen einsetzen können, aufgrund derer das Individuum noch weniger als zuvor Herr im eigenen Haus ist und Geschichte selbst zur Dispositionsmasse von keineswegs 
absichtslosen „Digital narrators" und deren „Digital storytelling“ wird (Alexander 2017). Dann steht aber nicht mehr nur der notwendige Schutz von Privatheit, der etwa alle archivalische Arbeit rechtlich bestimmt, auf dem Prüfstand. Sondern zugleich droht eine wesentliche Dimension der gesellschaftlichen Selbstbestimmung über das Erinnert-werden und Vergessen-werden verloren zu gehen. Was also ist zu tun bzw. zuvor gefragt: Was und wie ist theologisch zu denken?

\section{Erinnern und Vergessen in Zeiten digitaler Bildung}

Es mag eigenartig und sachfremd erscheinen, an dieser Stelle eine theologische Perspektive einzuspielen. Gleichwohl kann diese sowohl angesichts der Frage freier Selbstbestimmung wie auch einer weiterreichenden Erinnerungskultur für den Umgang mit den genannten Herausforderungen von orientierender Relevanz sein. Erinnern und Vergessen sind kulturell bedingte Menschheitskünste, die durch die lange europäische Geschichte hindurch auch erheblich von christlichen Menschen- und Gottesbildern mitgeprägt worden sind. Mit den Möglichkeiten und Formen digitaler Speicherung stellen sich die urmenschlichen und theologischen Fragen nach Selbstbestimmung über wesentliche Identitätsfragen (Lövheim 2013) in neuem, sozusagen disruptivem Gewand.

Zur Annäherung soll ein Schritt zurück zur gegenwärtigen bildungstheoretischen Debatte um die Erinnerungskultur gemacht werden, indem die oben aufgeworfenen Fragen auf digitale Formen von Holocaust-Erinnerung hin konkretisiert werden. Denn hier bilden sich in digitalen Zeiten wesentliche Herausforderungen des Umgangs mit der Frage von Speichern, Erinnern und auch Vergessen ab. Aus den folgenden Einblicken sollen zugleich wesentliche Einsichten zur Frage der - auch digital gesicherten - Verfügbarkeit individueller und kollektiver Identitäten gewonnen werden. Von dort aus wird im Schlussabschnitt aus theologischer Sicht auf die individuelle und kollektive existenzielle Dimension von Erinnern und Vergessen in digitalen Zeiten zurückzukommen sein.

Die schulische und damit auch die religionsbezogene Bildungspraxis der letzten rund 50 Jahre ist mindestens für den Kontext Deutschland vom Narrativ geprägt, dass Auschwitz sich nicht wiederhole (Adorno 1966). Dies verbindet sich mit einer in den vergangenen Jahrzehnten in schulischen Zusammenhängen etablierten Erinnerungskultur, welche stark auf die Schrecknisse des Holocaust als einem entscheidenden Grundpfeiler europäischer Erinnerungskultur Bezug nimmt. In Zeiten digitaler Speicherdyna- 
miken nimmt diese Erinnerungskultur neue Formen an, wirft aber zugleich drängende Fragen der Verfügbarkeit, Privatheit und auch der Würde der erinnerten Personen auf.

Dabei gilt grundsätzlich: Die eine Erinnerung an den Holocaust gibt es nicht. Dies zeigt sich eindrücklich im Roman "Monster" des israelischen Schriftstellers Yishai Sarid (Sarid 2019). Die Geschichte ist schnell erzählt: Der Protagonist, ein jüdischer Historiker, ist ausgewiesener Kenner der Vernichtungsmaschinerie des Holocaust und hat darüber erfolgreich promoviert. Die Zahlen der Opfer, die Logistik und Fakten der Auslöschung sind ihm bestens vertraut. Und so wird er im Auftrag der Gedenkstätte Yad Vashem zum Experten und führt Gruppen und Personen durch die europäischen Vernichtungslager. Doch alsbald taucht das Monster der Erinnerung auf. Im wahrsten Sinn des Wortes aus dem Boden kommen die Geister - und diese sind von ganz anderer Qualität als alle Zahlen und Fakten. Die historische Expertise bricht angesichts der sich dunkel manifestierenden Erinnerungskräfte geradezu über ihm und in ihm zusammen. Man kann diesen Roman so lesen: Holocaust-Erinnerung ist im Fluss, selbst wenn die äußeren Daten und Fakten weitgehend unbestritten sind. Weil sich die Zeiten und Umstände ändern, muss immer wieder neu erinnert werden und auch für das Vergessen ergeben sich immer wieder neue Herausforderungen.

Dieser metaphernreiche Roman steht pars pro toto für eine gegenwärtig intensiv geführte bildungstheoretische Debatte: Die inzwischen vierte Generation von Jugendlichen kann bekanntermaßen selbst nicht mehr auf unmittelbare Familiennarrative zurückgreifen. Auch die letzten Zeitzeugen sterben oder können keine Auskunft mehr geben (Gross and Stevick 2015). Aufgrund der kulturellen Pluralisierung der gegenwärtigen Jugendgeneration pluralisieren sich auch die Erinnerungsnarrative und -identitäten in erheblicher Weise: Ausgrenzungs-, Vertreibungs- oder Genoziderfahrungen sind längst nicht mehr exklusive Erfahrungen von jüdischen Menschen oder von damals ebenfalls der Vernichtung preisgegebenen anderen Minderheiten. Zudem sind in politischer Hinsicht in den vergangenen Jahren erhebliche Auseinandersetzungen um das Holocaust-Narrativ entstanden. Nationalistische und (wiederum!) identitäre, ethnische und auch bestimmte religiös-fundamentalistische Bewegungen erheben Anspruch auf „die eine wahre“ Deutung der Vergangenheit, also darauf, wer erinnert und wer vergessen werden soll - und dies in unverkennbarem Eigen- und Machtinteresse. Was soll erinnert werden? Wer entscheidet über die relevanten Erinnerungen? Wer bestimmt die Narrations-, Erinnerungs- und Vergessenspolitik? Was wird aus dem Monster und aus den Geistern der Erinnerung? 
Nun könnte man vermuten und hoffen, dass ja gerade die digitale Kultur es ermöglicht, diese Erinnerungen nicht nur wach, sondern tatsächlich anschaulich zu halten. Diesbezüglich liegen aus jüngster Zeit interessante Versuche vor, gerade im Rekurs auf digitale Speichermedien zu einer solchen kollektiven und kommunikativen Erinnerungskultur beizutragen. Im Bereich der Geschichtsdidaktik hat sich in den vergangenen Jahren eine intensive Debatte entwickelt, Erinnerung mittels digitaler Medien neu zu erschließen. Grundsätzlich werden die Recherchemöglichkeiten im digitalen Zeitalter dadurch erleichtert, dass etwa konkrete lokale Rechercheprojekte mit vergleichsweise geringem Aufwand möglich werden (vgl. Bernsen und Kerber 2017, Demantowski und Pallaske 2014; exemplarisch und mit Hinweisen auf einzelne Initiativen Pallaske 2015). Interessanterweise werden digitale Medien angesichts des Todes der letzten Zeitzeugen zu neuen Erinnerungsorten: Das Projekt „Papierblatt.de“ etwa versteht sich als Projekt gegen das Vergessen und gegen Antisemitismus. Es handelt sich dabei um ein frei zugängliches Online-Video-Archiv mit authentischen Zeitzeugenberichten in deutscher Sprache und zugleich um eine digitale Plattform für Unterricht und Forschung mit Suchfunktion, Materialsammlung und Unterrichtsentwürfen (vgl. Papierblatt 2016).

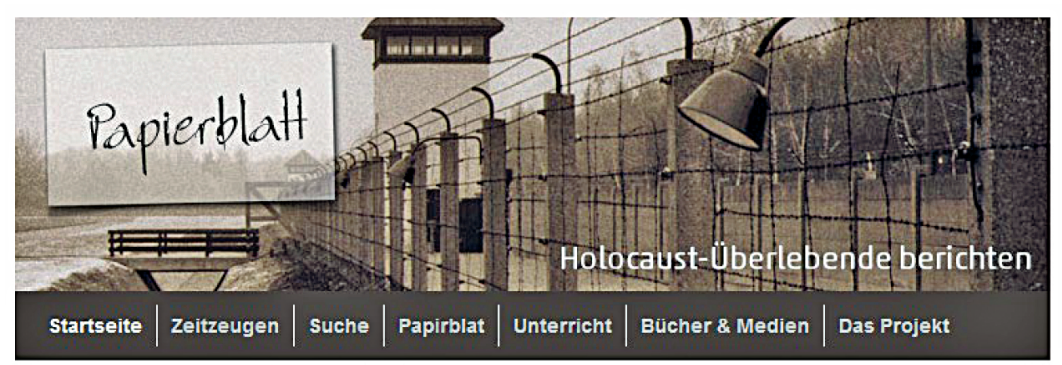

\section{Holocaust-Überlebende berichten}

Ein Video-Archiv gegen das Vergessen

Die digitalen Medien können aber auch selbst zu einer Art neuem digitalem Erinnerungsnarrativ werden, das bewusst eine Brücke zwischen Vergangenheit und Gegenwart schlägt. Dies gilt besonders eindrücklich, aber auch streitbar für das Projekt „@Eva-Stories“. Unter dem Titel „What if a Girl in the Holocaust had Instagram?" wird im Instagram-Modus das Leben der 13-jährigen Eva nachgezeichnet bzw. digital miterlebt (Eva Stories, 2019). 


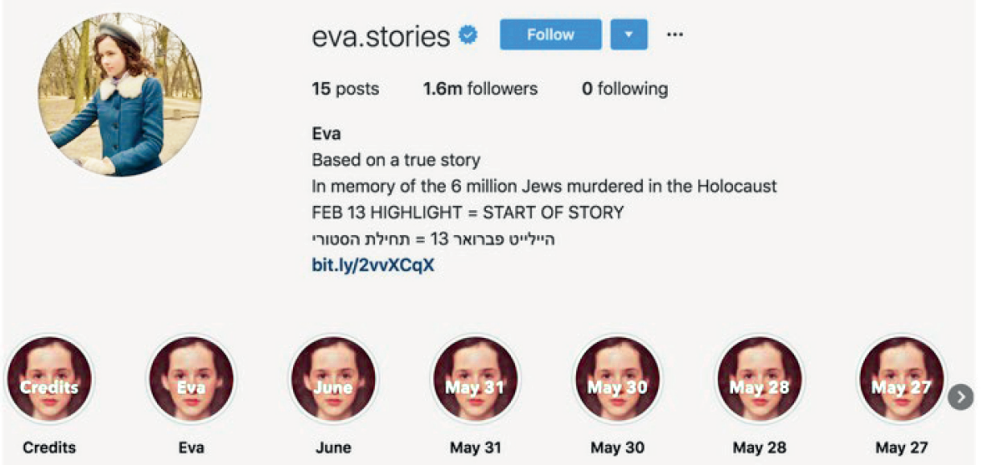

eva.stories on Instagram.

Die Idee der israelischen Filmemacher ist es, Jugendlichen vor dem Hintergrund ihres bevorzugten Mediums die damaligen Ereignisse anhand der Geschichte einer tatsächlich in Auschwitz ums Leben gekommenen Jugendlichen anschaulich zu machen.

Schließlich hat in jüngerer Zeit ein weiteres digitales Format für erhebliche Aufmerksamkeit und Irritation gesorgt: die sogenannte Gedenkstätten-Selfie-Kultur. Junge Menschen fotografieren sich etwa vor dem Eingangstor von Buchenwald, zwischen den Stelen des Berliner Denkmals der Ermordung der Juden, vor den Krematorien von Auschwitz. Darüber sind jeweils intensive Debatten entbrannt: Was passiert hier? Kommt dies einer Entweihung des Gedenkens und einer Entwürdigung der Opfer durch die Nachgeborenen gleich? Ist dies sozusagen im Nachhinein respektlos und menschenverachtend? Oder könnte es sein, dass junge Menschen genau eine solche Form der Erinnerung - und eben auch des Vergessens - pflegen, weil es ihrer Idee von bedeutsamer Selbstinszenierung und -repräsentation entspricht (vgl. Gojny, Kürzinger und Schwarz 2016)? Überschreiben sie mit diesem Selfiegebrauch die Dignität des Opfergedenkens oder machen sie dieses Gedenken möglicherweise besonders anschaulich? Anschaulich und höchst ambivalent ist in diesem Zusammenhang die deutsch-israelische Koproduktion „uploading-holocaust“, die aus einer filmischen Zusammenstellung von youtube-Videos israelischer Jugendlichen auf einer „Tour“ durch die Holocaust-Gedenkstätten besteht. 
Der israelische Satiriker Shahak Shapira hat im Jahr 2017 diese SelfieGedenkkultur kritisch in seinem Projekt namens „Yolocaust“ auf die Spitze getrieben (vgl. Yolocaust 2017), indem er die Selfies mit den realen Bildern der Vernichtung verbunden und diese Montagen an die Selfie-Macher selbst zurückgespielt hat (euronews 2017).

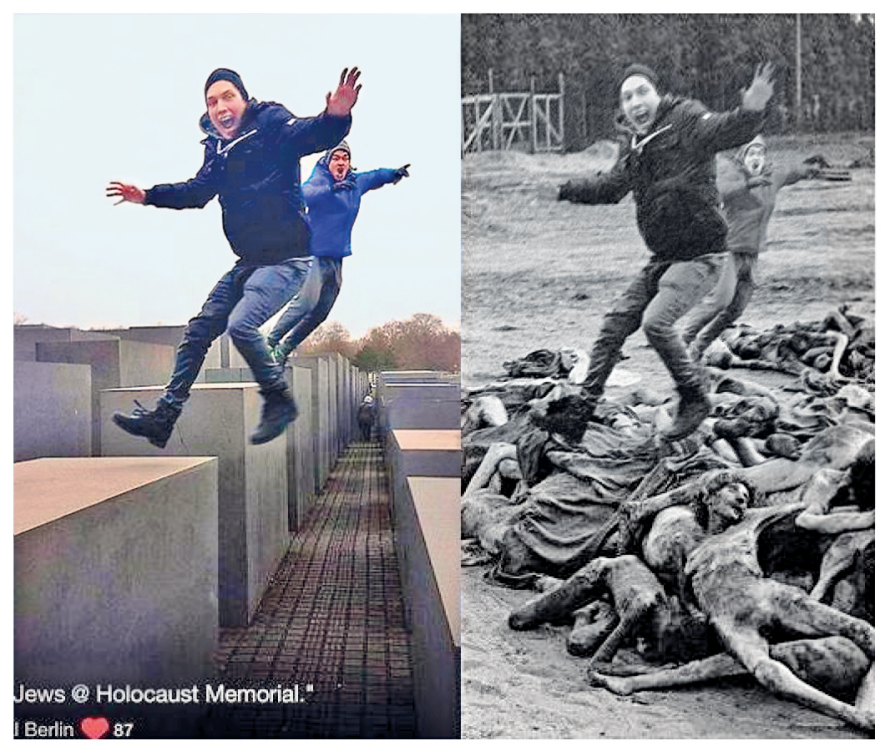

An diesen Beispielen wird erkennbar, dass die Frage der Erinnerung und des Vergessens unzähliger Opfer-Identitäten und der gesamten Geschichte des Grauens durch die Möglichkeiten digitaler Speicherung die anfangs aufgeführten Fragen nochmals in besonders dramatischer Weise aufwirft. Erinnerung wird festgehalten. Die didaktischen Möglichkeiten der digitalen Oral History sind beeindruckend. Aber können die digitalen Speicherund Erinnerungsformen das ersetzen, was „die Alten“ von Angesicht zu Angesicht erzählt haben? Und ist Erinnerung aufgrund ihrer Funktionalisierungsoffenheiten auch gefährlich (vgl. Körtner 2019, 148)?

Extrembeispiele zeigen klar, dass die Frage der Verfügbarkeit über erzählte Erinnerung gerade digital besonders leicht gemacht wird. Wie steht es in digitalen Zeiten um die Würde derer, die nicht mehr leben? Wer darf digital verfügen, wer darf vergessen werden, wer bleibt aus welchen Gründen in Erinnerung, wo wird anhand der digitalen Möglichkeiten über die Würde derer verfügt, die sich nicht mehr wehren können, wer wird aus 
bestimmten politischen Gründen eingeschlossen oder ausgeschlossen? Es drohen digitale Erinnerungen, die betroffene Menschen auf ihre damaligen Erfahrungen ein für alle Mal festlegen oder gar durch „Deep-fake“-Technologien in ein gänzlich anderes Licht stellen: Die Opfer bleiben Opfer. Die Täter bleiben Täter. Es droht damit nicht nur die Gefahr, dass Geschichte mit einem bestimmten Interesse erzählt wird. Sondern fast noch bedrohlicher ist eine weitere Konsequenz: „The greatest threat isn't that people will be deceived, but that they will come to regard everything as deception" (Schwartz 2018).

Und so ist noch einmal zugespitzt zu fragen: Haben Menschen und Schicksale gerade angesichts digitaler Speicherdynamiken ein Recht darauf $\mathrm{zu}$ vergessen bzw. vergessen zu werden? Diese Frage ist alles andere als unerheblich, insofern bisher gerade das „Nicht-vergessen“ der Opfer den Kern der Erinnerungskultur ausgemacht hat.

Nun hat Erinnerung aber als seine zweite Seite auch die Dimension des Vergessens: Erinnern und Vergessen sind „menschliche Eigenschaften, die weder gut noch schlecht sind, sondern beide gehören dazu, das Leben zu bewältigen" (so Jan Philipp Reemtsma nach Assmann 2018, 67). Schon das Vergessen selbst beinhaltet unterschiedliche Techniken: Das Löschen, das Zudecken, das Verbergen und Verdrängen, das Schweigen, das Überschreiben, das Ignorieren, das Neutralisieren, das Leugnen und auch das Verlieren. Die Dynamik des kulturellen Gedächtnisses umschließt also zentripetale Kräfte des Erinnerns und zentrifugale Kräfte des Vergessens. Es macht tatsächlich aus verschiedenen Gründen erheblichen kulturellen Sinn, manches bewusst zu vergessen (vgl. Butzer und Günter 2004; Dimbath und Heinlein 2014). Eine der Hauptfunktionen des Gedächtnisses besteht gerade im Vergessen, wodurch eine Selbstblockierung des Systems durch frühere Beobachtungen unterbunden wird. Hauptfunktion des Vergessens ist folglich das Freimachen von neuen Kapazitäten (vgl. Luhmann 1997). Plädiert wird dabei keineswegs für ein bewusstes Löschen von Erinnerung oder gar ein Überdecken oder Ignorieren in politisch-strategischer Absicht. Ein solches Vergessen wäre eine Waffe, eine aggressive und lautlose Form der Erhaltung von Macht, um den Schutz von Tätern und die Stabilisierung eines repressiven sozialen Klimas aufrecht zu erhalten (vgl. Assmann 2018, 67).

Vergessen hat vielmehr eine konstruktive und sogar therapeutische Funktion. Im Bild gesprochen: „Die Seite muss gelesen werden, bevor sie umgeblättert werden kann" (Assmann 2018, 65). Manches muss schlichtweg erst einmal gelesen werden, damit man überhaupt überleben und weiterleben kann. Manche Monster der Erinnerung sind nur dadurch zu bekämpfen, 
dass man sich ihnen stellt, um von dort aus weiter ins Leben schreiten zu können. Und vor allem gilt in diesem Zusammenhang, dass das Vergessen eben nicht der Würde der Einzelnen widerspricht, sondern gerade deren Würde bewahrt. Erinnerung kann deshalb nicht ohne das Vergessen gedacht werden.

Lässt sich dann aber in digitalen Zeiten überhaupt noch so etwas wie ein kollektives und kommunikatives Gedächtnis erzeugen oder pflegen oder im wahrsten Sinn des Wortes am Leben erhalten? Was passiert, wenn das Vergessen - technisch gesehen - kaum noch möglich ist und nichts mehr definitiv gelöscht werden kann? Drohen hier nicht ähnliche Speicherprobleme, wie sie anfangs angedeutet wurden, unter denen dann dasjenige der technischen Speicherung das weitaus Geringste ist?

So zeigt sich am konkreten, zugegebenermaßen extremen Beispiel der Holocaust-Erinnerung, dass das Aufrufen von Erinnerung sowie Prozesse des Vergessens gerade in diesem Fall bzw. in diesen Einzelfällen Aushandlungen voraussetzen und dabei immer die Würde desjenigen im Zentrum stehen muss, über dessen „Verbleib“ jeweils entschieden wird. Übrigens wurde diese Diskussion unter ganz analogen Voraussetzungen auch schon um die sogenannten Stolpersteine geführt - verbunden mit der Frage, ob damit nicht mit Füßen auf den Namen der Opfer „herumgetreten“ werde (Goebel 2010).

Damit stellt sich die weitergehende Frage danach, nach welchen inhaltlichen Kriterien erinnert und vergessen werden soll und kann. Erinnerung und Vergessen können jedenfalls in digitalen Zeiten nicht alleine aufgrund von technischen Möglichkeiten bestimmt werden, ebenso wenig wie sich ein gemeinsames kulturelles Gedächtnis allein dadurch einstellt, dass möglichst vieles gesammelt und gespeichert wird. Vielmehr bedarf es hier immer der persönlichen existenziellen Auseinandersetzung. Dafür lohnt es sich, nochmals auf die theologische Dimension zu sprechen zu kommen.

\section{Erinnern und Vergessen in theologischer Perspektive}

Im ersten Abschnitt kam bereits eine metaphernreiche theologische Semantik zum Tragen, indem etwa von „vergeben“, „neu anfangen können“, „unsterblich sein“ und sogar von der „Arche“ die Rede war. $\mathrm{Zu}$ prüfen und zu diskutieren ist, inwiefern eine theologische Perspektive auf das Speichern in digitalen Zeiten von erschließender Bedeutung sein kann. 
Erinnerungslernen ist in erheblicher Weise mit theologischen Perspektiven verbunden. Denn Religionen haben spezifische Erinnerungspotenziale, die erst und nur im Zusammenhang mit dem Gott-Mensch-Verhältnis ihren tieferen Sinn erhalten. Erinnerung ist mindestens in jüdischer und christlicher Hinsicht ein wesentliches Merkmal und ein Grundpfeiler des individuell-kollektiven religiösen Selbstverständnisses. Sie umfasst dabei die von der Gottesbeziehung her zu verstehenden Zeitdimensionen von Vergangenheit, Gegenwart und Zukunft (vgl. Boschki und Schlag 2015).

Um dies an einem Beispiel deutlich zu machen: Im Jahr 1996 hat der damalige israelische Staatspräsident Ezer Weizman im Deutschen Bundestag zur Erinnerung an den Holocaust die folgenden Worte gefunden: „Erst 150 Generationen sind seit der Feuersäule des Auszugs aus Ägypten bis zu den Rauchsäulen der Shoah vergangen. Und ich, geboren aus den Nachkommen Abrahams im Lande Abrahams, war überall mit dabei. Ich war ein Sklave in Ägypten und empfing die Thora am Berg Sinai, und zusammen mit Josua und Elijah überschritt ich den Jordan. Mit König David zog ich in Jerusalem ein, und mit Zedekiah wurde ich von dort ins Exil geführt. Ich habe Jerusalem an den Wassern zu Babel nicht vergessen, und als der Herr Zion heimführte, war ich unter den Träumenden, die Jerusalems Mauern errichteten. Ich habe gegen die Römer gekämpft und bin aus Spanien vertrieben worden. Ich wurde auf den Scheiterhaufen in Magenza, in Mainz, geschleppt, und habe die Thora im Jemen studiert. Ich habe meine Familie in Kischinev verloren und bin in Treblinka verbrannt worden. Ich habe im Warschauer Aufstand gekämpft und bin nach Eretz Israel gegangen, in mein Land, aus dem ich ins Exil geführt wurde, in dem ich geboren wurde, aus dem ich komme und in das ich zurückkehren werde" (Weizman 1996).

Die Frage nach der individuellen und kollektiven jüdischen Identität lässt sich - so kann man aus diesen Worten folgern - nur über die Zeiten hinweg und im Angesicht der Gottesbeziehung immer wieder neu formulieren. Dabei wird das Vergangene so erinnert, dass die frühere Geschichte in fast paradoxem Sinn zugleich erinnert und vergessen wird. Verwiesen werden kann hier etwa auf das jährliche Pessach-Fest und im Speziellen den Seder-Abend, wo das Vergangene mit den Speisen und dem Erzählen der Exodusgeschichte und der Pessach-Haggada-Geschichte jeweils neu und existenziell aktualisiert wird. Vergessen wird hier folglich nicht im Sinn der Überschreibung oder Negierung, sondern im Sinn der von Gott her auf Erlösung hin ausgerichteten Zukunft und damit der Verbindung von Schöpfung, Offenbarung und Erlösung über die Zeiten hinweg verstanden (vgl. Scholem 1988, 541). 
Insofern manifestiert sich die Glaubensidentität von Beginn an in der Suche nach dem anwesenden Gott oder bzw. und der Erfahrung des abwesenden Gottes. Der eigene Glaube lebt somit vom immer wieder zu vollziehenden, sich stets aktualisierenden, fragend-existenziellen, klagenden und lobenden Rückgriff auf die vergangene Geschichte Gottes mit seinen Menschen um der gegenwärtigen Neuvergewisserung willen.

Die Frage, warum Gott das Leid im Holocaust überhaupt zulassen konnte, ist folglich nicht ohne die theologische Arbeit an der Erinnerung und dem Vergessen zu denken. So geht etwa Hans Jonas vom Verzicht Gottes auf die Allmacht aus: Nicht, weil er nicht gewollt, sondern weil er nicht gekonnt habe, habe Gott in Auschwitz nicht eingegriffen. Der Grund dafür liege in der Struktur der Schöpfung. In seiner Allmacht habe Gott aus Liebe die Welt erschaffen, aber seit der Schöpfung sei er nicht mehr allmächtig, sondern teile seine Macht mit der Welt. Jonas bezieht sich dabei auf die kabbalistische Lehre von der Selbstentäußerung und der Selbsteinschränkung Gottes nach dem Akt der Schöpfung: „Nachdem er sich ganz in die werdende Welt hineingegeben hat, hat Gott nichts mehr zu geben: Jetzt ist es am Menschen, ihm zu geben“" (Jonas 1987, 47). Mit anderen Worten: Auf das menschliche Leid lässt sich keine auch nur annähernd rationale Antwort nach menschlichen Maßstäben geben, selbst wenn sich vieles politisch rekonstruieren und in Zahlen fassen lässt. Zugleich ist die Dimension des Vergessens von erheblicher Bedeutung: Aber Vergessen nicht im Sinn der Verdrängung, sondern des bewussten Durchdringens der Erinnerung auf die Eröffnung von neuen Lebensmöglichkeiten hin.

Damit wird deutlich, dass nach jüdischem und christlichem Verständnis die theologische Dimension der Erinnerung auch eine eminent anthropologische Dimension hat. Tatsächlich erinnert sich Gott nach jüdischer Vorstellung an den Menschen und bewahrt durch die Nennung des Namens die Würde jedes Einzelnen. Dahinter steht die religiöse Hoffnung, dass niemand vergessen werden soll und jeder mit seinem eigenen Namen für immer bei Gott eingeschrieben bleibt.

Vergessen im Sinn von Erlösung, in der sich neutestamentlich „in soteriologischer Hinsicht die eschatologische mit der präsentischen Dimension“ (Reinmuth 2020, 84) verbindet, nimmt als erfahrene und erhoffte Aufhebung des Leidens eine wichtige konstruktive und sogar therapeutische Funktion an: Auch biblisch gesprochen muss erst gelesen werden, bevor umgeblättert werden kann, damit überhaupt überlebt und weitergelebt werden kann. Ein solches Vergessen „von Gott her" widerspricht nicht der Würde der Einzelnen, sondern bewahrt gerade deren Würde: „Der verletzliche Mensch ist konstitutiv der erlösungsbedürftige Mensch" (Grözinger 
2020, 21). Gottes Gnade besteht folglich nicht nur in der Erinnerung, sondern auch in der Aufhebung von Schmerz, Leid und Klage, also in der Vergebung und Erlösung des Menschen und des ganzen Volkes Israel. Dies bedeutet gnadentheologisch, dass Gott „aufheben“ kann, was sich der Mensch hat zuschulden kommen lassen. Man mag dies etwa im Sprachbild nachgezeichnet finden, in dem zukunftsbezogen davon die Rede ist, dass die früheren Nöte vergessen werden und nicht mehr vor Gottes Augen stehen (Jes 65,16). Dieses Vergessen ist aber kein Ignorieren, sondern eine Form der Annahme des Menschen als Person und nicht aufgrund seiner Taten, Unterlassungen oder Boshaftigkeiten. Die jüdisch-christliche Hoffnung besteht darin, dass der Mensch bei Gott geborgen ist und damit keiner menschlichen Fremdbestimmung mehr unterliegt. Oder wie es Elie Wiesel sagt: „We must not see any person as an abstraction. Instead, we must see in every person a universe with its own secrets, with its own treasures, with its own sources of anguish, and with some measure of triumph" (Wiesel 1992, ix).

Von diesen Überlegungen der Unverfügbarkeit menschlicher Würde aus lassen sich Folgerungen für die gegenwärtigen digitalen Speicherpraktiken und deren Ambivalenzen ziehen: Theologische Aufgabe ist es, die digitalen Möglichkeiten des Speicherns von der Frage her, was den Menschen in seinem Wesen ausmacht, kritisch zu bedenken. Und dies mit der Prämisse, dass die Identität des Menschen unverfügbar ist und komplexer, als es ein Speichermedium je dokumentieren könnte. Theologisches Hauptkriterium für die Einschätzung digitaler Speichermedien ist, ob durch diese die Unverfügbarkeit des Wesens des Menschen gewahrt bleibt: Der Mensch soll darüber, was von ihm erinnert bzw. vergessen wird, selbst entscheiden und verfügen können. Dabei gilt theologisch, dass ein wesentlicher Unterschied zwischen menschlicher Speicherung und göttlichem Gedächtnis besteht, der nicht übersprungen werden kann und darf. Theologisch gesehen kann der einzelne Mensch darauf vertrauen, dass seine Zukunft nach seinem Ableben nicht davon abhängig ist, ob er oder sie virtuell noch präsent bleibt, und es muss Raum dafür bleiben, von den gespeicherten Erinnerungen auch erlöst werden zu können.

Es geht somit nicht nur in juristischer Hinsicht um die Frage der Selbstbestimmung des Menschen, sondern theologisch auch um die Anerkennung seiner geschöpflichen Würde. Es bedarf demzufolge gerade inmitten der Kultur der Digitalität eines möglichst großen Freiraumes für die Deutung und Erschließung individueller und kollektiver Geschichte. 
Abschließend gilt es, die verschiedenen Perspektiven nochmals im Sinn einer zukünftigen medienkritischen, theologisch pointierten Bildungs- und Erinnerungsarbeit zusammenzudenken.

\section{Folgerungen für eine persönlich und existentiell bedeutsame Bildungs- und Erinnerungskultur}

Wie anfangs gesagt, sind angesichts der gegenwärtigen digitalen Dynamiken erst einmal die notwendigen Fragen zu stellen. Gerade weil sich noch nicht absehen lässt, welche prometheischen, disruptiven Kräfte die digitalen Welten freisetzen werden, ist prophylaktische Wachsamkeit unbedingt erforderlich. Diese Fragen sind in ihrer Relevanz für eine kritische und zugleich zivilgesellschaftlich konstruktive Bildungs- und Erinnerungskultur kaum zu überschätzen. Es bedarf folglich einer kritischen Wahrnehmung in Hinsicht darauf, was in den verborgenen digitalen Welten mit den individuellen und kollektiven Identitätskonstruktionen geschieht und welche Autoritäten Anspruch darauf erheben dürfen.

Damit sich eine Gesellschaft über ihre eigenen Grundlagen im Klaren sein kann, muss sie sich in aller Offenheit ihrer eigenen Herkunftsgründe vergewissern können. Aber erinnernde Vergewisserung und vergewissernde Erinnerung können nicht von oben dekretiert oder gar „anonym“ gehalten werden. Diese lebensbedeutsamen Grundlagen gelangen nur dann ins aktive Bewusstsein der jeweiligen Gemeinschaft, wenn sie sowohl kollektiv wie kommunikativ immer wieder neu erinnert und in Erinnerung gehalten werden. Dies bedeutet aber zugleich, dass die jeweilige Erinnerung niemals objektiv festgesetzt werden kann, sondern immer wieder neuer Deutung bedarf. Denn die Bedingungen, unter denen erinnert wird, sind prinzipiell immer kontextueller Art und unterscheiden sich je nach biographischer Erfahrung, Herkunft oder Generation. Je nach Sprachfähigkeit und Reflexionsbereitschaft unterscheiden sich auch die kommunikativen Interaktionen deutlich voneinander. So bedarf es überall dort der kritischen Wachsamkeit und Aufmerksamkeit, wo versucht wird, kollektive Identitäten in ideologischem Sinn durch Formen einer digital verordneten Erinnerungskultur zu schaffen und damit zugleich bestimmte Exklusionen voranzutreiben.

Eine solche „Disziplinierung der Wahrnehmung“ (Zierold 2008, 186) verbindet sich zugleich mit einer möglichst frühen medienkritischen Bildung. Hier ist in besonderer Weise religiöse Bildung dazu aufgerufen, durch den Bezug auf ihre Kerngehalte für fundamentalistische Ausgrenzungsdynamiken zu sensibilisieren und so Mitmenschlichkeit zu befördern. Kollektive 
und kommunikative Erinnerungskulturen können gerade dadurch befördert werden, dass sie die Erinnerung an die memoria passionis (Metz 2006) als Teil einer jüdisch-christlich mitgeprägten Bildungs- und Erinnerungskultur lebendig halten (vgl. Forschungsgruppe REMEMBER 2020).

Bildungspraktisch bedeutet dies, bezogen auf die Frage der HolocaustErinnerung, nie nur das Opferkollektiv, sondern den je einzelnen Menschen als von Gott her erinnerten und erlösten Menschen wahrzunehmen. In diesem gelingenden Fall eröffnen digitale Speichermedien den Blick auf die Komplexität des Menschen vor Gott - und dies im Bewusstsein, dass das Ganze des Menschen und seiner Geschichte nie wirklich festgehalten werden kann.

Dies führt abschließend nochmals auf die Frage des kompetenten Umgangs mit den digitalen Medien zurück: Diese Kompetenz richtet sich nicht primär auf die Frage der Technik, sondern auf die Fähigkeit zu einer viel weitergehenden Einschätzung. Um nochmals Joseph Weizenbaum zu zitieren: „Wir müssen vom Menschen ausgehen, nicht vom Medium. Anders gesagt: Wir können heute nicht mehr erkennen, ob das Bild, das wir gerade auf dem Fernsehschirm sehen, ein reales oder ein hergestelltes, bearbeitetes ist. Wie sollen wir das entscheiden? Meistens fangen wir am falschen Ende an: Wenn wir nämlich befürchten, dass die Bilder, die bei uns ankommen, manipuliert oder verfälscht wurden, dann doch nicht, weil die Maschine dazu in der Lage ist, sondern weil wir es den Menschen, die sie bedienen, zutrauen. Also, wieder einmal: Die wichtigste Frage lautet, ob wir den Menschen vertrauen können“ (Weizenbaum 2006, 174f.). Die freie Entscheidung über das Erinnert- und Vergessen-werden gehört zum Wesen des Menschen, aber auch zur gesellschaftlichen Selbstverständigung über geschichtliche Ereignisse und Prozesse. Man sollte einstweilen mit Jaron Lanier ernsthaft überlegen, was man von sich selbst der digitalen Erinnerung preisgeben will und im Zweifelsfall eben nicht digital zu partizipieren, bestimmte Medien schlichtweg zu verlassen, um so wenige Spuren wie möglich zu hinterlassen (Lanier 2018).

Zugleich „wissen“ das Netz und seine Betreiber*innen natürlich um unseren Narzissmus, die Sehnsucht nach Anerkennung, Aufmerksamkeit sowie nach verlässlichen Beziehungs- und Netzwerkstrukturen - um unsere Sehnsucht nach Ewigkeit sowieso. Wie schwer digitale Enthaltsamkeit sein kann, mag man daran erahnen, dass Versuche der digitalen Askese oder gar der Widerstand gegen bestimmte autoritativ-algorithmische Speicherprinzipien ihrerseits mit nachhaltiger Speicherung rechnen müssen. Aber all dies kann aus besten theologischen Gründen kein Grund dafür sein, sich den 
digitalen Speicherdynamiken willenlos, klaglos oder gar selbstvergessen auszusetzen.

\section{Literaturverzeichnis}

Adorno, Theodor W. 1966. „Erziehung nach Auschwitz.“ In Erziehung zur Mündigkeit, Vorträge und Gespräche mit Hellmuth Becker 1959-1969, edited by Gerd Kadelbach, 92-109. Frankfurt am Main: Suhrkamp.

Alexander, Brian. 2017. The New Digital Storytelling. Creating Narratives with New Media, revised and updated $2^{\text {nd }}$ Edition. Santa Barbara: Praeger.

Arch Mission Foundation. 2020. Access date: 02.02.2020.

https://en.wikipedia.org/wiki/Arch_Mission_Foundation

Arendt, Hannah. 1994. Über die Revolution. 4. Auflage. München: Piper (On Revolution. New York: 1963).

Arte. 2018. Digital Black Hole. Ankündigungstext der arte-Sendung vom 5. Juli 2018. Access date:

03.10.2019. https://www.arte.tv/de/articles/tracks-digital-black-hole Assmann, Aleida. 2018. Formen des Vergessens. Bonn: BpB.

Bernsen, Daniel, and Kerber, Ulf, ed. 2017. Praxishandbuch Historisches Lernen und Medienbildung im digitalen Zeitalter. Opladen u.a.: Verlag Barbara Budrich.

Boschki, Reinhold, and Schlag, Thomas. 2015. „Zeit-Wege und Wege-Zeit der Tora - Chancen eines beziehungsorientierten Erinnerungslernens." Zeitschrift für Pädagogik und Theologie 67, no. 2, 145-154.

Brand, Stewart. 1999. "Escaping the digital dark age." Library Journal 124, no. 2: 46-48.

Butzer, Günter, and Günter, Manuela, ed. 2004. Kulturelles Vergessen. Medien - Rituale - Orte. Göttingen: Vandenhoeck \& Ruprecht.

Charta der Digitalen Grundrechte der Europäischen Union. 2018. Überarbeitete Fassung vom April 2018. Access date: 13.11.2019. https:/digitalchar ta.eu/wp-content/uploads/Digitale_Charta_320x468-FREITAG.pdf

Demantowski, Marko, and Pallaske, Christoph, ed. 2014. Geschichte lernen im digitalen Wandel. Berlin: De Gruyter.

Dimbath, Oliver, and Heinlein, Michael, ed. 2014. Die Sozialität des Erinnerns. Beiträge zur Arbeit an einer Theorie des sozialen Gedächtnisses. Wiesbaden: Springer VS. 
Euronews. 2017. Yolocaust: A satirist's challenge to Holocaust tourist behaviour. Access date: 11.03.2020. https://www.euronews.com/2017/01/19/ yolocaust-a-satirist-s-challenge-to-holocaust-tourist-behaviour

Eva Stories. 2019. Access date: 11.11.2019. https://www.instagram.com/eva .stories/?hl=en

Forschungsgruppe REMEMBER. 2020. Erinnerung an den Holocaust im Religionsunterricht. Empirische Einblicke und didaktische Impulse. Stuttgart: Kohlhammer.

Goebel, Anne. 2010. „Neue Diskussion über die ,Stolpersteine،“" Süddeutsche Zeitung, May 17, 2010.

https://www.sueddeutsche.de/muenchen/opfer-des-ns-terrors-neue-disku ssion-ueber-die-stolpersteine-1.677117

Gojny, Tanja, Kürzinger, Kathrin S., Schwarz, Susanne, ed. 2016. Selfie - I like it. Anthropologische und ethische Implikationen digitaler Selbstinszenierung. Stuttgart: Kohlhammer.

Grözinger, Albrecht. 2020. „,...uns aus dem Elend zu erlösen`. Gelebte (Erlösungs-)Religion zwischen Totalitarismus und individueller Freiheit.“ In Braucht der Mensch Erlösung?, edited by Christof Landmesser and Doris Hiller, 13-21. Leipzig: Evangelische Verlagsanstalt.

Gross, Zehavit, and Stevick, E. Doyle, ed. 2015. As the witnesses fall silent. 21st century Holocaust education in curriculum, policy and practice. Cham: Springer International.

Herzog, Stephanie. 2018. „Der digitale Nachlass und das Erbrecht. Universalsukzession gilt auch in der digitalen Welt - vorsorgende Rechtspflege stärken." AnwBl Online, 472-481. Access date: 11.12.2019. https://ww w.juris.de/jportal/portal/page/bsabprod.psml?showdoccase $=1 \& s \mathrm{st}=\mathrm{zs} \&$ doc.id=jzs-AnwBlOnline201806_005\&doc.part=S\&doc. price=0.0\#fo cuspoint

Jonas, Hans. 1987. Der Gottesbegriff nach Auschwitz. Eine jüdische Stimme. Frankfurt am Main: Suhrkamp.

Körtner, Ulrich H.J. 2019. „Geschichte und Eschatologie.“ In Geschichte und Gerechtigkeit, edited by Aleida Assmann, Jan Assmann, Oliver Rathkolb, 148-151. Wien: Lit-Verlag.

Lanier, Jaron. 2018. Ten Arguments For Deleting Your Social Media Accounts Right Now. London: Bodley Head.

Lövheim, Mia. 2013. "Identity." In Digital Religion. Understanding Religious Practice in New Media Worlds, ed. by Heidi A. Campbell, 41-56. Routledge: London and New York. 
Luhmann, Niklas. 1997. Die Gesellschaft der Gesellschaft. Frankfurt am Main: Suhrkamp.

McLuhan, Marshall. 1962. The Gutenberg Galaxy. The making of typographic man. Toronto: University of Toronto Press.

Metz, Johann Baptist. 2006. Memoria passionis. Ein provozierendes Gedächtnis in pluralistischer Gesellschaft. Freiburg im Breisgau: Herder.

Newton, Casey O.J. "Speak, Memory. When her best friend died, she rebuilt him using artificial intelligence." Access date: 11.04.2020. https:// www.theverge.com/a/luka-artificial-intelligence-memorial-roman-mazure nko-bot

Nora, Pierre. 1989. "Between Memory and History: Les Lieux de Mémoire." In Representations 26, Special Issue: Memory and CounterMemory, 7-24.

Orwell, George. 1949. 1984. London: Secker \& Warburg.

Pallaske, Christoph. 2015. „Digitales Geschichtslernen zum Holocaust | Praxisbeispiele | Workshop Berlin 27. Januar 2015." Access date: 11.11.2019. https://historischdenken.hypotheses.org/2715

Papierblatt. Holocaust-Überlebende berichten. Ein Projekt der MORIJA GmbH in Kooperation mit ZEDAKAH e.V. und dem Schuldekanat Calw-Nagold | Neuenbürg. Zugriff 9.11.2019. https://www.papierblatt.de/

Reinmuth, Eckart. 2020. „Erlösung. Neutestamentliche Perspektiven.“ In Braucht der Mensch Erlösung?, edited by Christof Landmesser and Doris Hiller, 61-85. Leipzig: Evangelische Verlagsanstalt.

Sarid, Yishai. 2019. Monster. Zürich: Kein \& Aber Verlag.

Scholem, Gerschom. 1988. „Franz Rosenzweig und sein Buch ,Der Stern der Erlösung." In: Franz Rosenzweig. Der Stern der Erlösung, 525-549. Frankfurt am Main: Suhrkamp.

Schwartz, Oscar. 2018. "You thought fake news was bad? Deep fakes are where truth goes to die." The Guardian, April 12, 2018. Access date: 04.04.2020.

https://www.theguardian.com/technology/2018/nov/12/deep-fakes-fake -news-truth

Uploading Holocaust. 2016. gebrueder beetz filmproduktion. Access date: 03.02.2020.

https://www.youtube.com/watch?v=Q4z6w3EiWIk)

Weizenbaum, Joseph. 2006. Inseln der Vernunft im Cyberstrom? Auswege aus der programmierten Gesellschaft. Bonn: BpB. 
Weizman, Ezer. 1996. „Staatsbesuch des Präsidenten des Staates Israel vom 14. bis 17. Januar 1996 - Besuch im Deutschen Bundestag - Rede des Präsidenten des Staates Israel." Access date: 11.03.2020.

https://www.bundesregierung.de/breg-de/service/bulletin/staatsbesuch-d es-praesidenten-des-staates-israel-vom-14-bis-17-januar-1996-besuch-im -deutschen-bundestag-rede-des-praesidenten-des-staates-israel-805848

Wiesel, Elie. 1992. "Foreword." In The Nazi Doctors and the Nuremberg Code. Human Rights in Human Eperimentation, edited by Annas, George J. and Grodin, Michael A., vii-ix, New York \& Oxford: Oxford University Press.

Yolocaust. 2017. By Shahak Shapira. Access date: 19.10.2019. https://yoloca ust.de/

Zierold, Martin. 2008. Gesellschaftliche Erinnerung: Eine medienkulturwissenschaftliche Perspektive. Berlin: De Gruyter. 